\title{
A Neutrosophic Approach to Edge Detection Using Ant Colony Optimization
}

\author{
Gulpi Qorik Oktagalu Pratamasunu ${ }^{1}$ and Olief Ilmandira Ratu Farisi ${ }^{2}$
}

\author{
${ }^{1}$ Department of Informatics, University of Nurul Jadid, Probolinggo, Indonesia \\ ${ }^{2}$ Department of Mathematics Education, University of Nurul Jadid, Probolinggo, Indonesia
}

\begin{abstract}
Neutrosophy theory has been widely used in solving problems involving uncertainty, especially in dealing with problems on noisy images. A new probabilistic rule to reduce the probability of noisy pixels becoming edges candidate is presented. This research proposed a new edge detection method on a digital image using Ant Colony Optimization (ACO) based on the neutrosophic set of gradient magnitude. Experimental results demonstrate the superior performance of the proposed approach on both the noise-free images and the images with different levels of noise. Based on the experimental results on the noise-free images, the proposed method has better performance than the other methods based on the visual quality and the value of the figure of merit. Evaluation of test images with different levels of noise illustrates the robustness of the proposed method on noisy images.
\end{abstract}

Keywords: Edge Detection, Ant Colony Optimization, Neutrosophic Set, Gradient Magnitude.

\section{Introduction}

One of the important stages in digital image processing applications is edge detection. In the medical field (detection of brain tumors and cancer), the results of edge detection are the basis for determining the decision of the analysis. So it requires the high accuracy of the edge detection algorithm. Therefore, finding a better algorithm to detect image edges is important because it is one of the key techniques in image processing and analysis.

The edge detection algorithm based on the gradient values, Sobel, Prewitt, Canny, etc, is widely used in image segmentation applications [1]. In this approach, edges can be defined as a change in the intensity of an image. The common approach is to calculate the gradient of the image and find the local optimum to detect edges. This approach can detect edges quickly, but it is very sensitive to noise.

Another approach that can be used is by using heuristic methods. Agrawal et al. [2] implemented ant colony optimization for edge detection which was outperformed edge detection using Sobel and Canny. Tian et al. [3] researched edge detection on a digital image using Ant Colony Optimization based on the Ant Colony System. This method gave better results with fewer disconnected edges than the method that implemented the Ant System. Unfortunately, the concept of searching in ACO relies on the values of the pixel's gradient information from each local ant. The ants can trap in a local optima situation and never visit some pixels which causes broken edges, especially in the presence of noise.

Some researchers are adding new information to guide ants to explore more edges and avoid local optima situations. Verma and Sharma [4] proposed a new approach to calculate the heuristic function of ACO by using the law of universal gravity. Rahebi et al. [5] combined ACO and genetic algorithm to avoid ants trapped in local optima and find the shortest paths. Zhang et al. [6] proposed the combination of gradient and relative difference of statistical means to suppress noise. Liantoni et al. [7] distributed ants adaptively based on the calculation of gradient analysis.

In this paper, a novel approach on edge detection using ACO based on neutrosophy theory is proposed. Neutrosophy has been widely used in solving problems involving uncertainty, especially in digital image processing problems [8]-[10]. Some researchers have conducted edge detection algorithms based on neutrosophy set [11]-[13]. According to the researchers,

Received: May 28 ${ }^{\text {th }}, 2021$. Accepted: August $26^{\text {th }}, 2021$ DOI: 10.15676/ijeei.2021.13.3.9 
the use of neutrosophic theory in edge detection algorithms yields good results on noisy images. Hence, to improve the robustness of ACO edge detection on noisy images, neutrosophic information is calculated as the new additional information that will be used along with pheromone and heuristic information of ACO to reduce the probability of noisy pixels becoming edges candidate.

\section{Basic Definition}

A. Neutrosophic Set

Neutrosophic set is a generalization of the classical set based on a branch of philosophy, namely Neutrosophy, which considers the origin, nature, theory, concept, event, proposition, or entity, its negation, and neutrality [14]. In neutrosophic sets, a set $S$ is divided into three subsets: entity $\langle S\rangle$, its negation $\langle$ anti $-S\rangle$, and $\langle n e u t-S\rangle$ which is not $\langle S\rangle$ nor $\langle$ anti $-S\rangle$. The subset $\langle S\rangle,\langle a n t i-S\rangle$, and $\langle n e u t-S\rangle$ represent the truth, false, and indeterminacy set, respectively. There is no stadard restriction $[0,1]$ on $\langle S\rangle,\langle$ anti $-S\rangle$, and $\langle$ neut $-S\rangle$. It allows inconsistency-tolerant and incomplete information which can be characterized in a neutrosophic set [15]. This makes the neutrosophic sets a good choice to deal with uncertainty problems in image processing, especially for edge detection in digital images.

Neutrosophic set has been widely used to overcome noise in the image and has been successfully applied in several problems regarding noise segmentation. In 2009, [8] proposed this theory in the case of solving segmentation problems on noisy images using $\gamma$-means clustering. In 2010, [9] proposed the use of this theory with the watershed method for segmenting noisy images. In 2014, [10] proposed the image thresholding algorithm based on a neutrosophic similarity score.

\section{B. Gradient Magnitude}

In mathematics, the gradient is one of the operators in vector calculus which is useful in finding changes in direction and speed in scalars. In an image, the gradient is formed by gradual discoloration [16]. For two-dimensional images, the application of gradients can be found by using spatial derivatives

$$
\left[\begin{array}{ll}
f_{x} & f_{y}
\end{array}\right]=\left[\begin{array}{ll}
\frac{d f}{d x} & \frac{d f}{d y}
\end{array}\right]
$$

where $f_{x}=\frac{d f}{d x}$ is derivative of $f$ with respect to $x$ and $f_{y}=\frac{d f}{d y}$ is derivative $f$ with respect to $y$.

Gradient magnitude is the magnitude or strength of a gradient. The edge point can be searched by finding the local maxima of the magnitude of the gradient. The greater the gradient magnitude, the greater the chance to find the edge of the image. The gradient magnitude $\Delta f$ defined as

$$
\|\Delta f\|=\sqrt{f_{x}^{2}+f_{y}^{2}}
$$

\section{Ant Colony Optimization}

Ant Colony Optimization (ACO) is a heuristic algorithm introduced by Marco Dorigo [17] to find the shortest path. This algorithm adopts the behavior of ant colonies in finding food sources. Ants leave substances called pheromones to communicate with their colonies. Ants tend to choose paths with a lot of pheromones. Pheromones sustain evaporation due to the air. This basic idea was then developed to solve a wider numerical problem, one of which was for edge detection.

ACO for image edge detection was proposed in [3]. To detect the edge of an image, the pixel is assumed to be a node. Ant moves from a point to edge pixels. Edge pixels are the path 
that ants must visit. In the selection process of pixels, ants consider several things, such as heuristic information $(\eta)$ in the form of local variation of the image's intensity value and pheromone trail $(\tau)$.

At the initialization process, there are three stages including ant's placement, pheromone initialization, and calculation of heuristic information. First, $K$-ants are distributed randomly on an $M_{1} \times M_{2}$ image. The initial values for each element of the pheromone matrix $\tau^{(0)}$ are set to be constant $\tau_{\text {init }}$. Heuristic information to detect edges is derived by the local statistics at the position of pixel- $(i, j)$ as

$$
\begin{aligned}
\eta_{i, j}= & \frac{V_{C}\left(I_{i, j}\right)}{\sum_{i=1}^{M_{1}} \sum_{j=1}^{M_{2}} V_{C}\left(I_{i, j}\right)} \\
V_{C}\left(I_{i, j}\right)= & f\left(\left|I_{i-2, j-1}-I_{i+2, j+1}\right|+\left|I_{i-2, j+1}-I_{i+2, j-1}\right|+\left|I_{i-1, j-2}-I_{i+1, j+2}\right|+\left|I_{i-1, j-1}-I_{i+1, j+1}\right|\right. \\
& \left.+\left|I_{i-1, j}-I_{i+1, j}\right|+\left|I_{i-1, j+1}-I_{i+1, j-1}\right|+\left|I_{i-1, j+2}-I_{i+1, j-2}\right|+\left|I_{i, j-1}-I_{i, j+1}\right|\right)
\end{aligned}
$$

where $V_{C}\left(I_{i, j}\right)$ is a function of a local group of pixel-c (clique) and depends on the variation of the image's intensity values of the pixel-c. There are four mathematical expressions to determine the function $f[2]$.

The next step is the construction process. At this stage, each ant moves $L$-movement steps in one iteration. The probability of ants move from pixel- $(p, q)$ to pixel- $(i, j)$ is using the proportional transition rule which is defined as

$$
P_{(p, q),(i, j)}^{(n)}=\frac{\left[\tau_{i, j}^{(n-1)}\right]^{\alpha} \eta_{i, j}^{\beta}}{\sum_{(i, j) \in \Omega_{(p, q)}}\left[\tau_{i, j}^{(n-1)}\right]^{\alpha} \eta_{i, j}^{\beta}}
$$

where $\tau_{i, j}^{(n-1)}$ is the trail of pheromone on pixel- $(i, j)$ and $\Omega_{(p, q)}$ is the neighborhood of pixel$(p, q)$, with $\alpha$ is the factor that influences the pheromones and $\beta$ is the factor that influences heuristic information $\eta_{i, j}[18]$.

After each ant moves from one pixel to another pixel, a local update of pheromones is carried out for each movement step. This pheromone update aims to reduce the concentration of pheromones on the visited pixel. The local update function in pheromones is defined as

$$
\tau_{i, j}^{(t-1)}(\text { new })=\left\{\begin{array}{ll}
(1-\varphi) \tau_{i, j}^{(t-1)}(\text { old })+\varphi \eta_{i, j}, & \text { if pixel- } J \text { is visited by ant }-k \\
\tau_{i, j}^{(t-1)}(\text { old }), & \text { otherwise }
\end{array} ;\right.
$$

with $\tau_{i, j}$ being pheromone on the pixel- $(i, j)$ and $\varphi$ is evaporation rate where $0<\varphi \leq 1$.

After all of the ants have passed one iteration, a global pheromone update is carried out. The function of updating global pheromones is defined as

$$
\tau^{(n)}=(1-\rho) \tau^{(n-1)}+\rho \tau^{(0)}
$$

where $\rho$ is the pheromone decay coefficient where $0<\rho \leq 1$. 
To determine whether a pixel is an edge pixel or not, a threshold $T$ for the final matrix $\tau^{(N)}$ is applied as a binary decision. The first step on the decision process is the initialization of $T^{(0)}$ which is defined as

$$
T^{(0)}=\frac{\sum_{i=1}^{M_{1}} \sum_{j=1}^{M_{2}} \tau_{i, j}^{(N)}}{M_{1} M_{2}}
$$

for $l=0$ iteration. The second step is separation the matrix $\tau^{(N)}$ into two classes. The first class is for $\tau$ whose value less than $T^{(l)}$, the second class if for the rest. Then, calculate the mean of the two classes by using

$$
\begin{gathered}
m_{L}^{(l)}=\frac{\sum_{i=1}^{M_{1}} \sum_{j=1}^{M_{2}} g_{T^{(l)}}^{L} \tau_{i, j}^{(N)}}{\sum_{i=1}^{M_{1}} \sum_{j=1}^{M_{2}} h_{T^{(l)}}^{L} \tau_{i, j}^{(N)}} \\
m_{U}^{(l)}=\frac{\sum_{i=1}^{M_{1}} \sum_{j=1}^{M_{2}} g_{T^{U}}^{U} \tau_{i, j}^{(N)}}{\sum_{i=1}^{M_{1}} \sum_{j=1}^{M_{2}} h_{T^{(l)}}^{U} \tau_{i, j}^{(N)}}
\end{gathered}
$$

where

$$
\begin{aligned}
& g_{T^{(l)}}^{L}(x)= \begin{cases}x, & \text { if } x \leq T^{(l)} \\
0, & \text { otherwise }\end{cases} \\
& h_{T^{(l)}}^{L}(x)= \begin{cases}x, \text { if } x \leq T^{(l)} \\
0, \text { otherwise }\end{cases} \\
& g_{T^{U}(l)}^{U}(x)= \begin{cases}x, \text { if } x \geq T^{(l)} \\
0, \text { otherwise }\end{cases} \\
& h_{T^{U}(l)}^{U}(x)= \begin{cases}x, \text { if } x \geq T^{(l)} \\
0, \text { otherwise }\end{cases}
\end{aligned}
$$

Then, update the threshold for the next iteration by using

$$
T^{(l)}=\frac{m_{L}^{(l)}+m_{U}^{(l)}}{2}
$$

If $\left|T^{(l)}-T^{(n-1)}\right| \geq \varepsilon$ then do the separation of the matrix $\tau^{(N)}$. Otherwise, the iteration process is terminated. If $\tau_{i, j}^{(N)} \geq T^{(0)}$, then the pixel- $(i, j)$ is an edge; otherwise, it is not.

\section{Proposed Method}

In this paper, we proposed a new approach to optimize ant movement in ACO for image edge detection. Standard ACO is used to distribute ants randomly over the image and move the ants based on pheromone and local variation of the image. This may cause an imbalance ant's distribution and can lead to local optima situation especially if noise present in the image. 


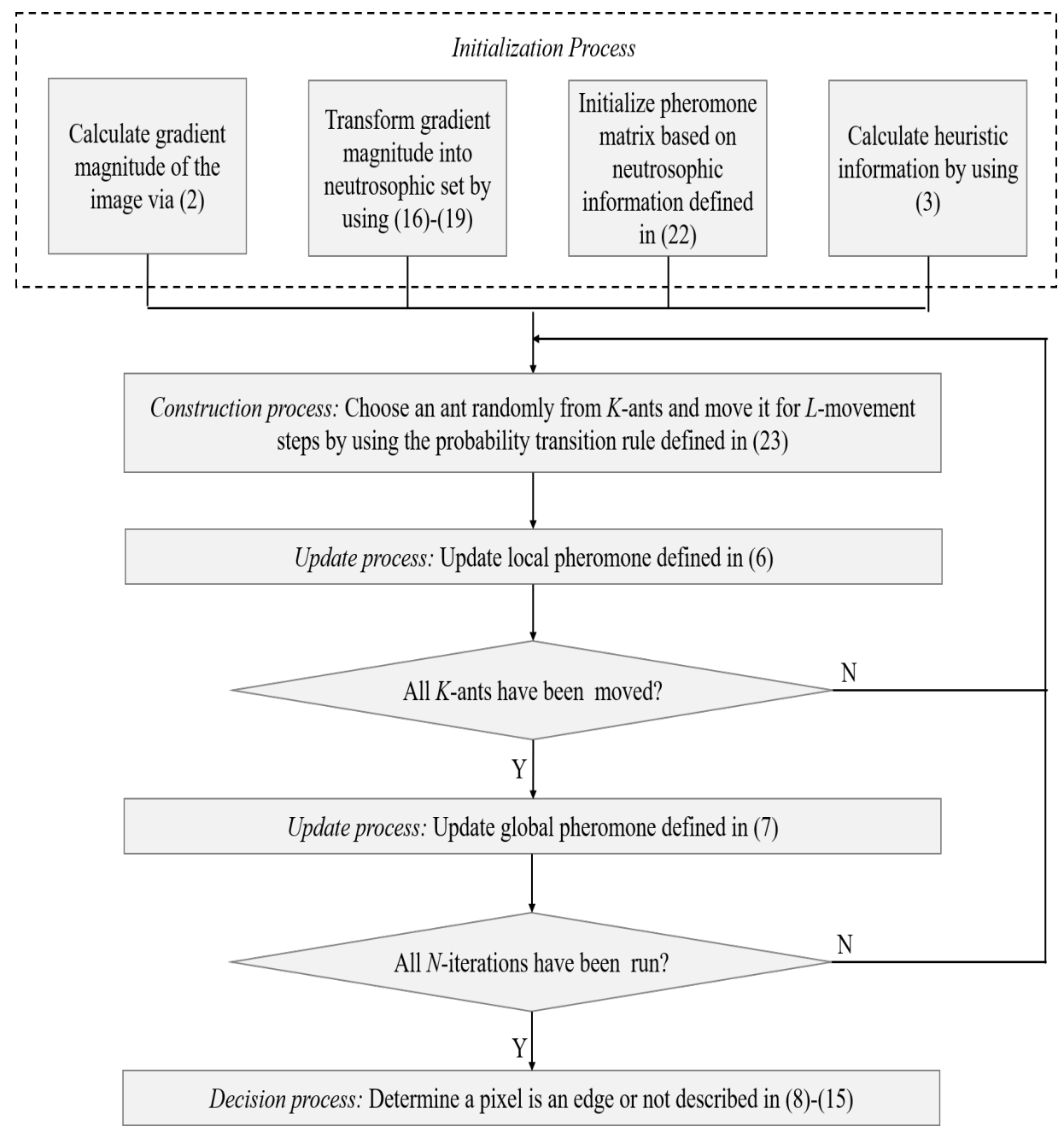

Figure 1. A summary of the implementation The Robust ACO Based On Neutrosphic Set of Gradient Magnitude

Our proposed method intended to use the gradient magnitude of the image as additional information for ant's movement calculation, as edges are assumed to be high gradient pixels [19]. However, most gradient-based edge detection algorithms like Sobel, Prewitt, Canny, etc. are sensitive to image noise that can lead to low performance of those methods. To overcome this problem, we use neutrosophic theory to construct a neutrosophic set of gradient magnitude as the additional information to ant's movement calculation. This way, we can reduce the probability of pixels that have high indeterminacy becoming edges candidates.

The first step of this proposed method is the initialization process of a neutrosophic set of gradient magnitude, initial pheromone matrix, and heuristic information. Then construct a pheromone matrix by iteratively performing construction and update process for $\mathrm{N}$ iterations. Finally, determine the edge using the decision process. A summary of the implementation of the proposed method is presented in Figure 1 and each of these processes is presented in detail as follows, respectively.

At the initialization process, the gradient magnitude of the image is calculated and transformed into a neutrosophic set. In this paper, the gradient magnitude is calculated by using the Sobel operator as shown in Figure 2. Neutrosophic set of gradient magnitude is described 
as three membership sets, namely $T, I$, and $F$. Pixel $\mathrm{P}(i, j)$ in the image domain transformed into a neutrosophic domain set and denoted by $P_{N S}(i, j)=\{T(i, j), I(i, j), F(i, j)\}$ which each represents an edge set of pixels, an indeterminate pixel set, and a set of non-edge pixels and is defined as

\begin{tabular}{|l|l|l|}
\hline-1 & 0 & +1 \\
\hline-2 & 0 & +2 \\
\hline-1 & 0 & +1 \\
\hline
\end{tabular}

$x$ filter

\begin{tabular}{|c|c|c|}
\hline+1 & +2 & +1 \\
\hline 0 & 0 & 0 \\
\hline-1 & -2 & -1 \\
\hline
\end{tabular}

y filter

Figure 2. Operator Sobel

$$
\begin{gathered}
T(i, j)=\frac{\nabla f(i, j)-\nabla f_{\text {min }}}{\nabla f_{\text {max }}-\nabla f_{\text {min }}} \\
I(i, j)=\frac{\delta(i, j)-\delta_{\text {min }}}{\delta_{\text {max }}-\delta_{\text {min }}} \\
\delta(i, j)=|\nabla f(i, j)-\nabla \bar{f}(i, j)| \\
F(i, j)=\frac{\nabla \bar{f}_{\text {max }}-\nabla \bar{f}(i, j)}{\nabla \bar{f}_{\text {max }}-\nabla \bar{f}_{\text {min }}}
\end{gathered}
$$

where $\nabla f$ is gradient magnitude, $\nabla \bar{f}$ is gradient magnitude after mean filter processing, and $\delta(i, j)$ is the absolute value of the difference in gradient magnitude between original image at pixel- $(i, j)$ and the pixel- $(i, j)$ after the mean filter processing. Mean filter processing is known as a simple and intuitive technique to suppress noise and smooth the images. The mean filter eliminates noise by replacing it using its surroundings.

To reduce the probability of pixels that are noisy pixels becoming edges candidate, the value of $\lambda$ (neutrosophic information) is calculated from $T, I$, and $F$ as the new additional information that will be used along with pheromone and heuristic information. By assuming pixels that have a high value of $I$ is pixels with high indeterminacy whether it's edge pixels or not, the value of $\lambda$ is defined as

$$
\lambda=T-I+(1-F)+T I-T F
$$

The initialization process comprises defining the number of ants $(K)$, ant's movement-steps per iteration $(L)$, the number of iterations in the construction process $(N)$, pheromone evaporation rate, pheromone decay coefficient and the weighting factor for pheromone, heuristic information, and neutrosophic information. Heuristic information and pheromone initialization are also conducted within this process. Heuristic information is derived from a local variation of pixel intensity. In this paper, heuristic information is calculated by using (2) where the function $f$ of $V_{C}\left(I_{i, j}\right)$ determined by

$$
f(x)=a x, \text { for } x \geq 0
$$

while initial pheromone for each pixel is uniformly distributed between 0 and 1 in ACO standard, the proposed method calculates the initial pheromone based on the value of neutrosophic information which is defined as 


$$
\tau^{(0)}=\lambda \tau_{\text {init }}
$$

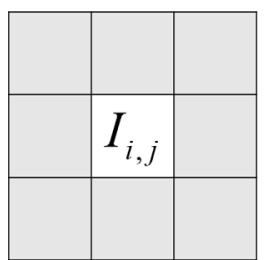

Figure 3. The 8-neighborhood connectivity

The second step of this proposed method is the construction process. At this stage, there are $K$-ants are randomly assigned to an $M_{1} \times M_{2}$ image. Each ant moves $L$-movement steps in one iteration for $N$-construction process. An ant moves from the pixel $(p, q)$ to its neighborhood pixel $(i, j)$ based on the new transition probability rule that is defined as

$$
P_{(p, q),(i, j)}^{(n)}=\frac{\left[\tau_{i, j}^{(n-1)}\right]^{\alpha} \eta_{i, j}^{\beta} \lambda_{i, j}^{\gamma}}{\sum_{(i, j) \in \Omega_{(p, q)}}\left[\tau_{i, j}^{(t-1)}\right]^{\alpha} \eta_{i, j}^{\beta} \lambda_{i, j}^{\gamma}}
$$

where $\tau_{i, j}^{(t-1)}, \eta_{i, j}, \Omega_{(p, q)} \alpha$ and $\beta$ as defined in (5). The $\lambda_{i, j}$ is the neutrosophic information and $\gamma$ is the weighting factor that influences neutrosophic information. The allowed range of ants to move and visit its neighborhood for $\Omega_{(p, q)}$ is determined by using the 8-connectivity neighborhood as shown in Figure 3.

When an ant moves from a pixel to another pixel, the pheromone will be updated using the local pheromone update defined in (6). After all of the ants complete their $L$-movement steps, a global pheromone update is carried out for each iteration as defined in (7). After $N$ iterations have been run, a decision is made whether a pixel is an edge or not based on the fitness values. In this paper, we used the same steps of the decision process as the ACO standard described in (8)-(15).

\section{Experimental Results}

A variety of images with different noise levels, Noise-free, Gaussian noise, and Salt \& Pepper noise, are employed to evaluate the performance of the proposed method. The proposed method (N-ACO) is compared with the existing ACO standard (S-ACO) for the edge detection algorithm. In addition, the proposed method is also compared with another three gradient-based edge detection algorithms, Sobel, Prewitt, and Canny. Furthermore, various parameters of the proposed method are set according to Table 1, based on several experiments that are not reported here.

To test the performance of the proposed method, eight images such as Checkboard, Camera, House, Lena, Pepper, Branch, Scissor, and Track, are used. The manually generated ground truth images have been defined for each original image. Figure 4 shows the original images and their ground truth in $128 \times 128$ resolution.

At first, the proposed method is evaluated on the original images without noise. The comparison of the results from noise-free images is shown in Figure 5. Then, the original images are added with Gaussian noise with a 0.5 mean and 0.01 variation. Figure 6 shows the comparison of the results from the original images with Gaussian noise. Lastly, the original images are added with Salt \& Pepper noise with 0.1 noise density. The results of the original images with salt $\&$ pepper noise are shown in Figure 7. 
Gulpi Qorik Oktagalu Pratamasunu, et al.

Table 1. Parameters of the proposed method

\begin{tabular}{|c|c|}
\hline Parameter & Parameter value \\
\hline$K$ & $\sqrt{M_{1} M_{2}}$ \\
\hline$\tau_{\text {init }}$ & 0.01 \\
\hline$\alpha$ & 1 \\
\hline$\beta$ & 1 \\
\hline$\gamma$ & 1 \\
\hline$a$ & 1 \\
\hline$\varphi$ & 0.0001 \\
\hline$\rho$ & 0.0001 \\
\hline$\varepsilon$ & 0.1 \\
\hline$L$ & 40 \\
\hline$N$ & 4 \\
\hline
\end{tabular}

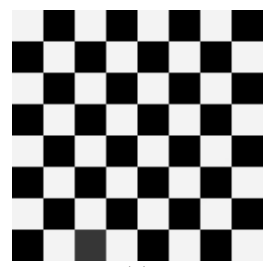

(a)

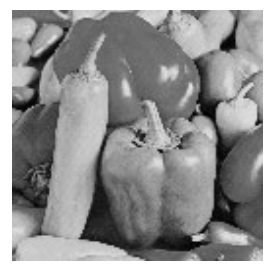

(e)

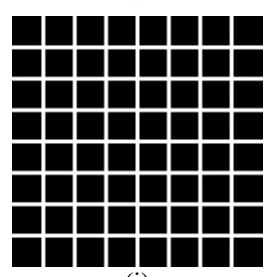

(i)

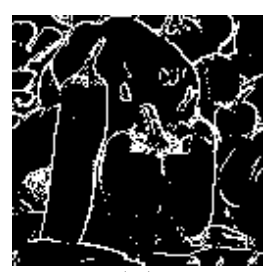

(m)

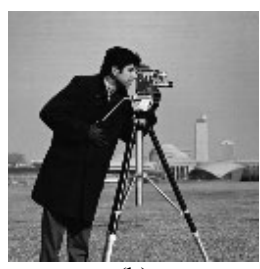

(b)

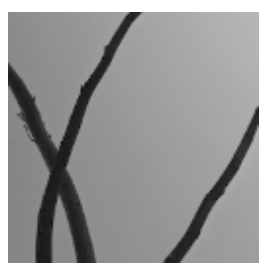

(f)

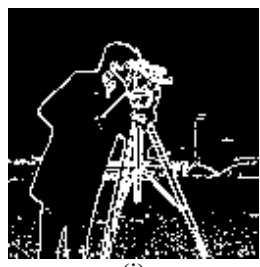

(j)

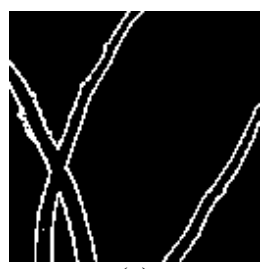

(n)

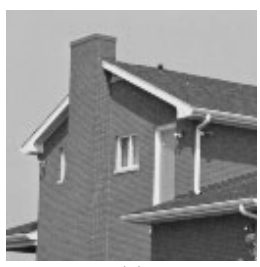

(c)

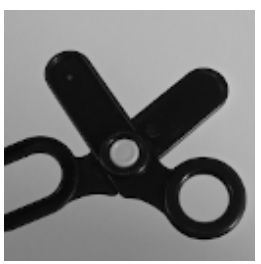

(g)

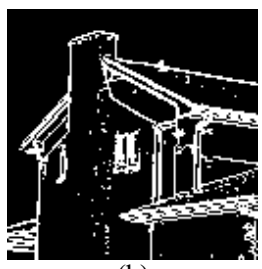

(k)

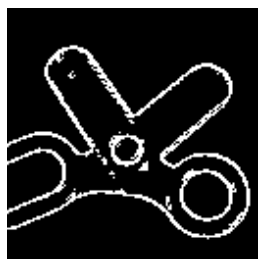

(o)

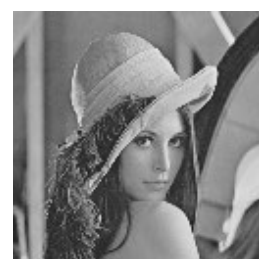

(d)

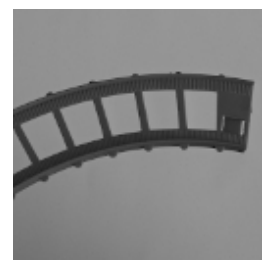

(h)

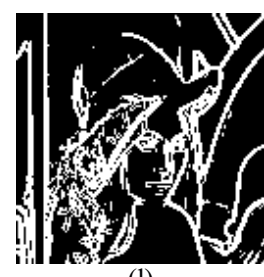

(1)

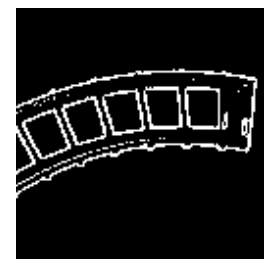

(p)

Figure 4. (a)-(h) The original image; (i)-(p) Ground truth of the original images, respectively 
Sobel
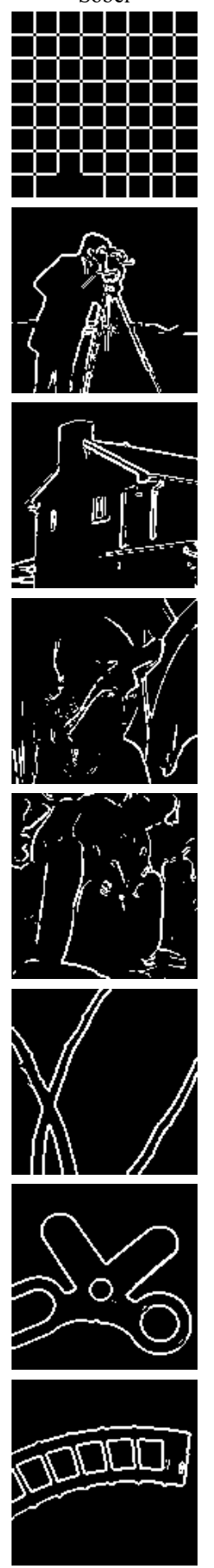

Prewitt
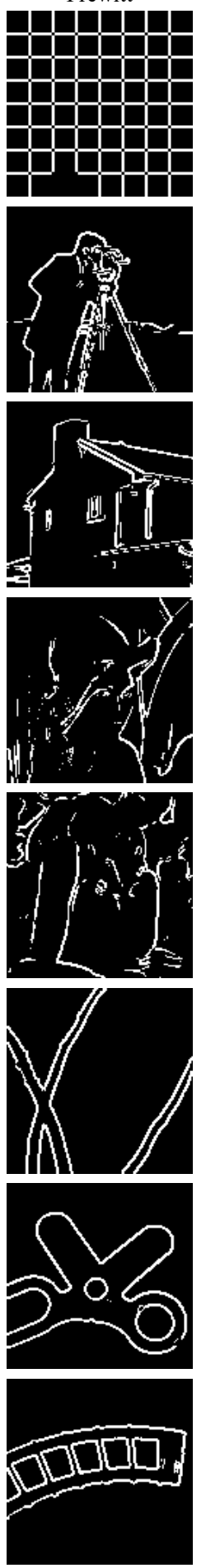

Canny
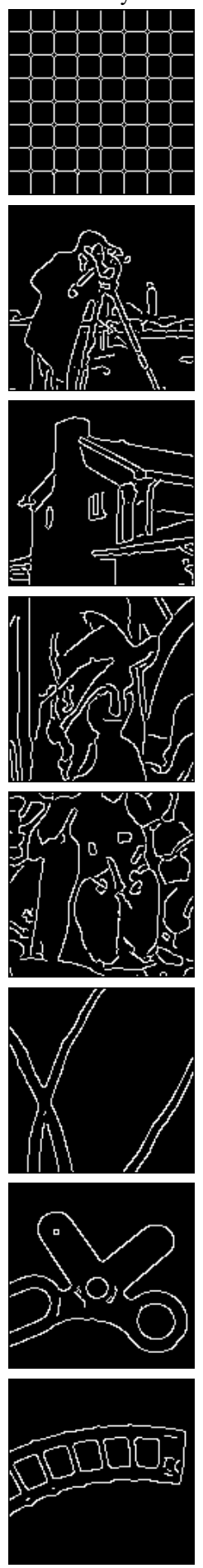

S-ACO
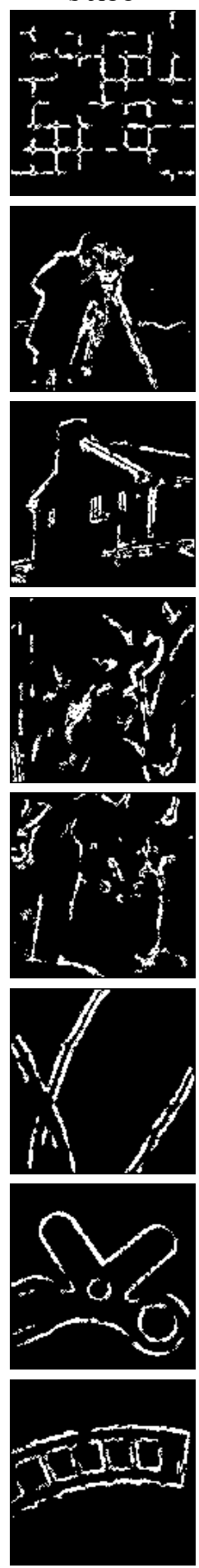

$\mathrm{N}-\mathrm{ACO}$
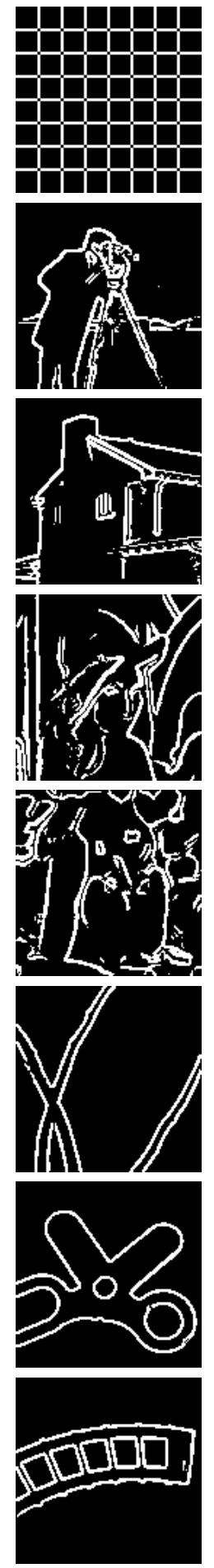

Figure 5. Edge detection comparison results on noise-free images

shown in Figure 5, the proposed method yields better results than the ACO standard, in terms of the visual quality of the extracted edge information on noise-free images. It showed 
Sobel
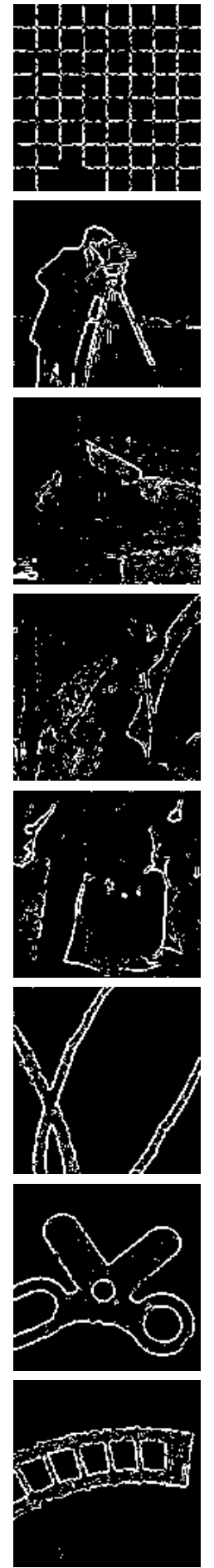

Prewitt
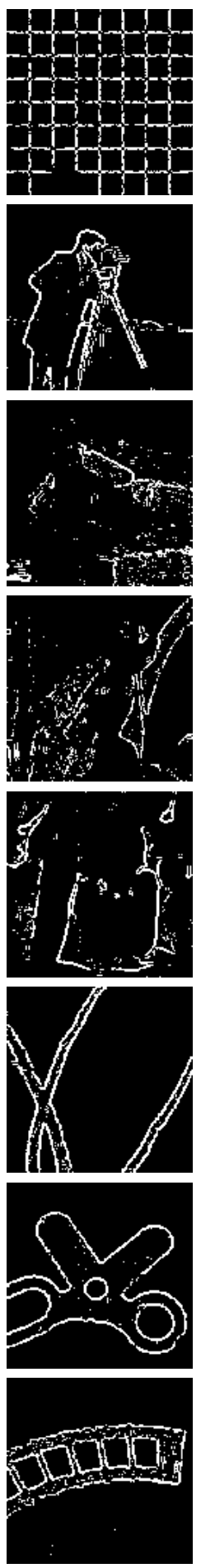

Canny
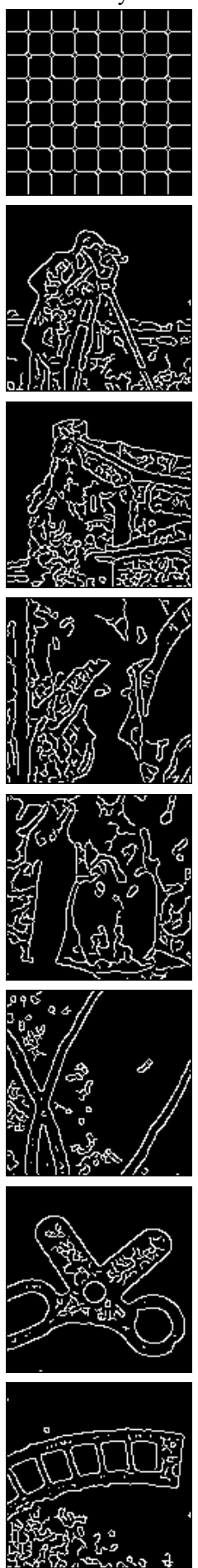

$\mathrm{S}-\mathrm{ACO}$
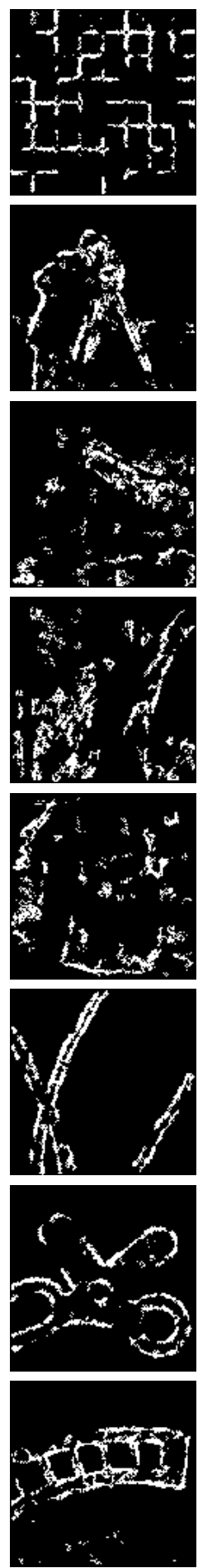

$\mathrm{N}-\mathrm{ACO}$
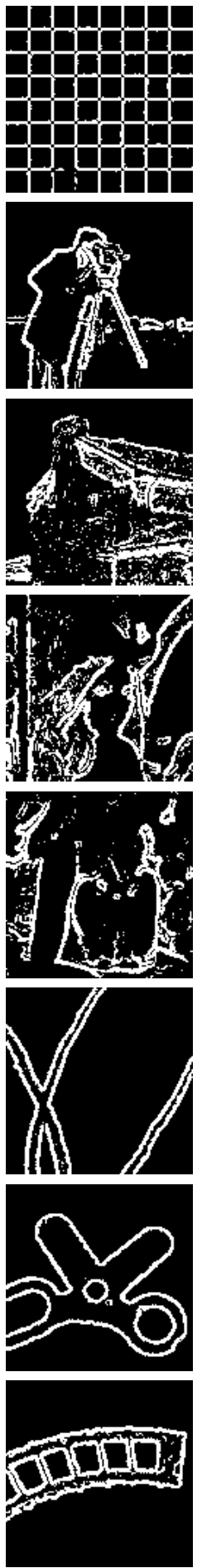

Figure 6. Edge detection comparison results on Gaussian noise images

that the usage of neutrosophic information in the initial pheromone and probabilistic transition rule of ant's movement can guide ants to discover more true edges than the existing method 


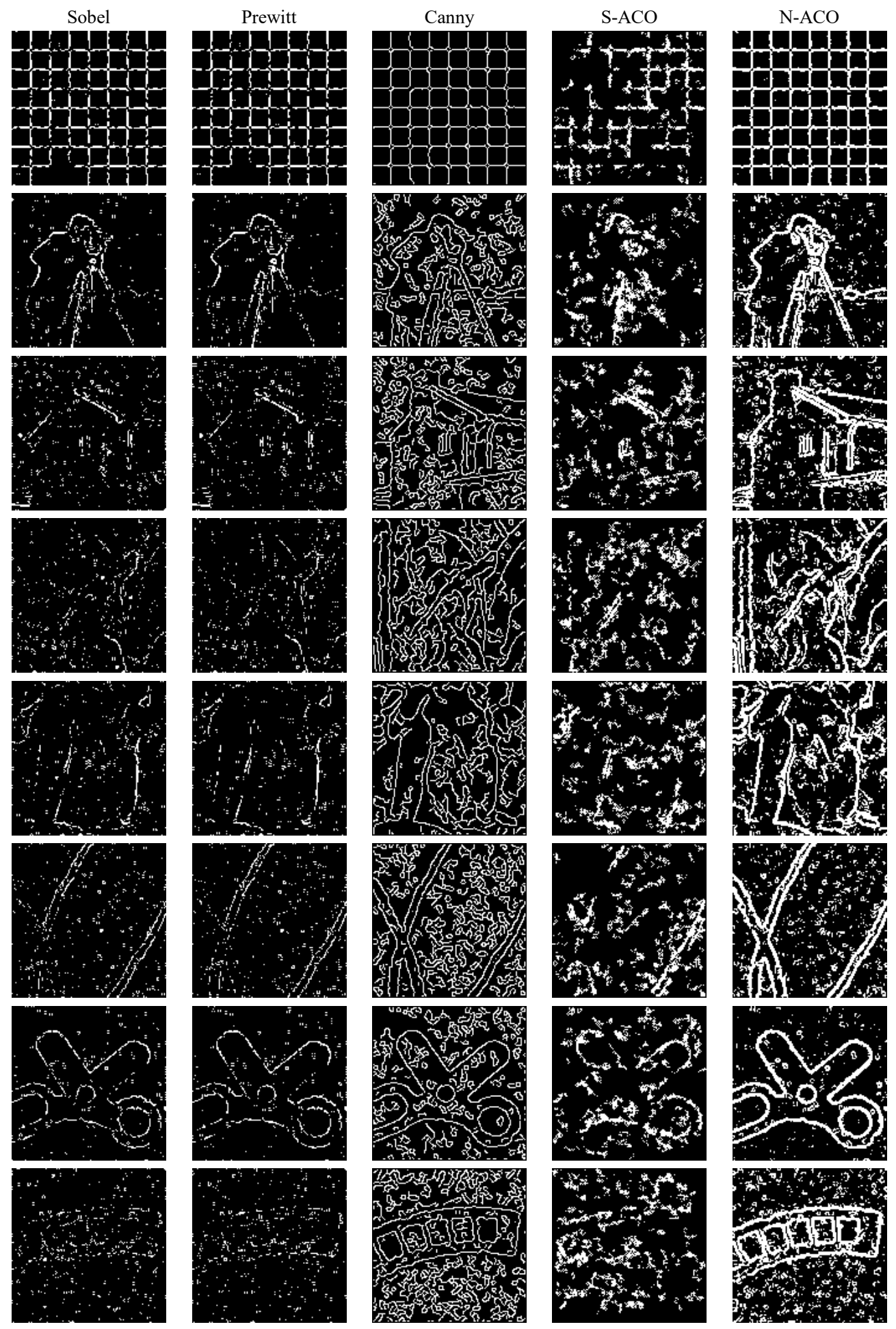

Figure 7. Edge detection comparison results on Salt \& Pepper noise images

that is only affected by pheromone and heuristic information. Figure 5 also showed that the proposed method produces results that have fewer disconnected edges than the results 
generated by other methods, especially for the Checkboard image.

From Figure 6 and 7, it can be seen that the proposed method performs well and achieves very good results, with few missing edges and false edges on noisy images. The detection performances of other methods are affected by noise and a lot of edges are missing and disconnected, especially for the Salt \& Pepper noise. As shown in Figure 6 and 7, the proposed method and Canny still maintained the shape of the object even in the noisy images. However, there are many false edges and noise in the result of Canny. This demonstrates the robustness of the proposed method in the presence of noise.

The performance of the N-ACO is quantitatively compared to other methods by using the figure of merit (FOM) [20]. This is done by comparing the ground truth images with the corresponding images provided by the five different methods. The formula to calculate the FOM is defined as

$$
F O M=\frac{1}{\max \left(N_{1}, N_{A}\right)} \sum_{k=1}^{N_{A}} \frac{1}{1+\omega d^{2}(k)}
$$

where $N_{1}$ and $N_{A}$ is the number of detected edge pixels and the number of actual edge pixels, respectively. Distance $d(k)$ is the distance from the $k$-th actual edge to the closest detected edge pixels. A constant $\omega$ is scaling constants and set to $1 / 9$ [15]. This FOM value determines the quality of edge detection, the greater the FOM value indicates the better the edge detection results.

Table 2. Comparison of FOM on original images among edge detection algorithms

\begin{tabular}{|l|c|c|c|c|c|}
\hline \multirow{2}{*}{\multicolumn{1}{|c|}{ Image }} & \multicolumn{5}{c|}{ Edge Detection Algorithm } \\
\cline { 2 - 6 } & Sobel & Prewitt & Canny & S-ACO & N-ACO \\
\hline Checkboard & 91.31 & 91.31 & 47.60 & 35.24 & 93.90 \\
\hline Camera & 44.67 & 44.63 & 39.81 & 35.67 & 52.82 \\
\hline House & 42.34 & 42.42 & 39.73 & 35.77 & 54.11 \\
\hline Lena & 27.57 & 28.10 & 35.09 & 31.82 & 67.10 \\
\hline Pepper & 39.81 & 38.76 & 45.33 & 33.27 & 65.18 \\
\hline Branch & 84.14 & 86.57 & 61.86 & 67.22 & 92.40 \\
\hline Scissor & 71.62 & 73.69 & 51.33 & 57.46 & 82.18 \\
\hline Track & 78.24 & 79.18 & 58.75 & 60.58 & 88.29 \\
\hline Average (\%) & 59.96 & 60.58 & 47.44 & 44.63 & 74.50 \\
\hline
\end{tabular}

Table 3. Comparison of FOM on Gaussian noise images among edge detection algorithms

\begin{tabular}{|l|c|c|c|c|c|}
\hline \multirow{2}{*}{\multicolumn{1}{|c|}{ Image }} & \multicolumn{5}{|c|}{ Edge Detection Algorithm } \\
\cline { 2 - 6 } & Sobel & Prewitt & Canny & S-ACO & N-ACO \\
\hline Checkboard & 59.99 & 65.10 & 48.91 & 37.46 & 92.95 \\
\hline Camera & 38.17 & 37.90 & 40.47 & 31.29 & 52.16 \\
\hline House & 18.72 & 18.84 & 33.50 & 19.51 & 52.44 \\
\hline Lena & 21.27 & 21.03 & 30.67 & 26.31 & 57.57 \\
\hline Pepper & 28.98 & 29.29 & 39.42 & 25.47 & 49.06 \\
\hline Branch & 76.06 & 78.88 & 59.61 & 60.98 & 94.61 \\
\hline Scissor & 61.95 & 64.89 & 52.13 & 51.88 & 85.96 \\
\hline Track & 63.27 & 65.31 & 55.52 & 53.28 & 91.65 \\
\hline Average (\%) & 46.05 & 47.65 & 45.03 & 38.27 & 72.05 \\
\hline
\end{tabular}


Table 4. Comparison of FOM on Salt\&Pepper noise images among edge detection algorithms

\begin{tabular}{|l|c|c|c|c|c|}
\hline \multirow{2}{*}{\multicolumn{1}{|c|}{ Image }} & \multicolumn{5}{|c|}{ Edge Detection Algorithm } \\
\cline { 2 - 6 } & Sobel & Prewitt & Canny & S-ACO & N-ACO \\
\hline Checkboard & 65.90 & 65.22 & 47.85 & 42.76 & 93.80 \\
\hline Camera & 21.64 & 21.74 & 39.40 & 35.71 & 60.82 \\
\hline House & 13.81 & 14.75 & 39.38 & 29.24 & 65.83 \\
\hline Lena & 9.89 & 9.45 & 34.13 & 23.61 & 67.75 \\
\hline Pepper & 17.24 & 17.28 & 41.35 & 31.56 & 68.14 \\
\hline Branch & 29.02 & 30.76 & 57.55 & 40.13 & 95.78 \\
\hline Scissor & 35.17 & 36.56 & 49.44 & 41.73 & 88.90 \\
\hline Track & 16.24 & 16.83 & 54.64 & 29.19 & 88.43 \\
\hline Average (\%) & 26.11 & 26.57 & 45.47 & 34.24 & 78.68 \\
\hline
\end{tabular}

Tables 2, 3, and 4 show the comparison of the FOM values for the detection results on original images, images with Gaussian noise, and images with Salt \& Pepper noise, respectively. Based on Tables 2, 3, and 4, all the detection results provided by the proposed method have the highest FOM. It can be seen that the gap of FOM values between the proposed method and others is greater on detection results of a noisy image than the detection results of original images. It indicates that the proposed method's performance is more robust in the presence of noise than the other methods.

\section{Conclusion}

This research proposed a new edge detection method on a digital image using Ant Colony Optimization (ACO) based on the neutrosophic set of gradient magnitude. Each pixel in the image is described as three memberships, namely edge pixels, non-edge pixels, and indeterminate pixels, which are determined by the gradient magnitude of the image. The value of neutrosophic information is calculated as the new additional information that will be used along with pheromone and heuristic information to reduce the probability of noisy pixels becoming edges candidate. To evaluate the performance of the proposed method, the results of the proposed method are compared with ACO standard and the gradient-based edge detection methods, Sobel, Prewitt, and Canny, on the images with different levels of noise, such as noise-free, Gaussian noise, and Salt \& Pepper noise. Based on the experimental results on the noise-free images, the proposed method has better performance than the other methods based on the visual quality and the value of the figure of merit. Evaluation of test images with different levels of noise illustrates the robustness of the proposed method on noisy images.

\section{References}

[1] D. Ziou and S. Tabbone, "Edge detection techniques: An overview," Int. J. Pattern Recognit. Image Anal., vol. 4, pp. 537-559, 1998.

[2] P. Agrawal, S. Kaur, H. Kaur, and A. Dhiman, "Analysis and Synthesis of an Ant Colony Optimization Technique for Image Edge Detection," in 2012 International Conference on Computing Sciences, 2012, pp. 127-131, doi: 10.1109/ICCS.2012.14.

[3] J. Tian, W. Yu, and S. Xie, "An ant colony optimization algorithm for image edge detection," in 2008 IEEE Congress on Evolutionary Computation (IEEE World Congress on Computational Intelligence), 2008, pp. 751-756, doi: 10.1109/CEC.2008.4630880.

[4] O. P. Verma and R. Sharma, "An optimal edge detection using universal law of gravity and ant colony algorithm," in 2011 World Congress on Information and Communication Technologies, 2011, pp. 507-511, doi: 10.1109/WICT.2011.6141297.

[5] J. Rahebi, Z. Elmi, A. Farzam nia, and K. Shayan, "Digital image edge detection using an ant colony optimization based on genetic algorithm," in 2010 IEEE Conference on Cybernetics and Intelligent Systems, 2010, pp. 145-149, doi: 
10.1109/ICCIS.2010.5518567.

[6] J. Zhang, K. He, X. Zheng, and J. Zhou, "An Ant Colony Optimization Algorithm for Image Edge Detection," in 2010 International Conference on Artificial Intelligence and Computational Intelligence, 2010, vol. 2, pp. 215-219, doi: 10.1109/AICI.2010.167.

[7] F. Liantoni, K. C. Kirana, and T. H. Muliawati, "Adaptive Ant Colony Optimization Based Gradient for Edge Detection," J. Ilmu Komput. dan Inf., vol. 7, no. 2, pp. 76-82, Aug. 2014, doi: 10.21609/jiki.v7i2.260.

[8] Y. Guo and H. D. Cheng, "New neutrosophic approach to image segmentation," Pattern Recognit., vol. 42, no. 5, pp. 587-595, 2009, doi: https://doi.org/10.1016/j.patcog.2008.10.002.

[9] M. Zhang, L. Zhang, and H. D. Cheng, "A neutrosophic approach to image segmentation based on watershed method," Signal Processing, vol. 90, no. 5, pp. 1510-1517, 2010, doi: 10.1016/j.sigpro.2009.10.021.

[10] Y. Guo, A. Şengür, and J. Ye, "A novel image thresholding algorithm based on neutrosophic similarity score," Measurement, vol. 58, no. 1, pp. 175-186, Nov. 2014, doi: 10.1016/j.compeleceng.2014.04.020.

[11] E. SERT and D. AVCI, "A new edge detection approach via neutrosophy based on maximum norm entropy," Expert Syst. Appl., vol. 115, pp. 499-511, Jan. 2019, doi: 10.1016/j.eswa.2018.08.019.

[12] E. Rashno and B. Minaei-Bidgoli, "Boundary points handling for image edge detection based on Neutrosophic set," in 2019 IEEE 5th Conference on Knowledge Based Engineering and Innovation, KBEI 2019, Feb. 2019, pp. 886-890, doi: 10.1109/KBEI.2019.8734912.

[13] P. Arulpandy and M. Trinita Pricilla, "Salt and pepper noise reduction and edge detection algorithm based on neutrosophic logic," Comput. Sci., vol. 21, no. 2 SE-Articles, Apr. 2020, doi: 10.7494/csci.2020.21.2.3438.

[14] F. Smarandache, "A Unifying Field in Logics: Neutrosophic Logic.," Philosophy. American Research Press, pp. 1-141, 1999, [Online]. Available: http://cogprints.org/1919/.

[15] F. G. Lupiáñez, “On Neutrosophic Sets and Topology,” Procedia Comput. Sci., vol. 120, pp. 975-982, Jan. 2017, doi: 10.1016/j.procs.2018.01.090.

[16] R. C. Gonzalez and R. E. Woods, Digital Image Processing (3rd Edition). Upper Saddle River, N.J.: Prentice Hall, 2008.

[17] M. Dorigo, V. Maniezzo, and A. Colorni, "Ant system: Optimization by a colony of cooperating agents," IEEE Trans. Syst. Man, Cybern. Part B Cybern., vol. 26, no. 1, pp. 29-41, 1996, doi: 10.1109/3477.484436.

[18] M. Dorigo, M. Birattari, and T. St, "Ant Colony Optimization,” no. November, 2006.

[19] C. Gupta and S. Gupta, "Edge Detection of an Image based on Ant Colony Optimization Technique," 2013. Accessed: May 28, 2021. [Online]. Available: www.ijsr.net.

[20] W. K. Pratt, Digital Image Processing. Nueva York, EUA : Wiley, 1991. 


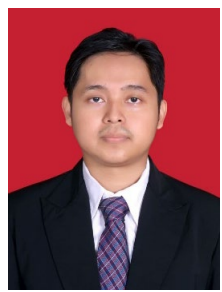

Gulpi Qorik Oktagalu Pratamasunu received the bachelor's degree in Informatics Education Universitas Negeri Malang (ID) and the master's degree majoring in Informatics at Institut Teknologi Sepuluh Nopember (ID). $\mathrm{He}$ is currently an Assistant Professor at Department of Informatics University of Nurul Jadid (ID). His research interests are in image processing, computer vision, and game development.

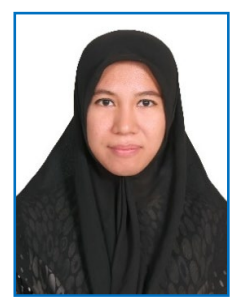

Olief Ilmandira Ratu Farisi is a lecturer at Department of Mathematics Education University of Nurul Jadid (ID). She received a master's degree majoring in Mathematics at Institut Teknologi Sepuluh Nopember (ID) and a bachelor's degree at Mathematics Education Universitas Negeri Malang (ID). Her research interest are in optimization, graph theory, and development of instructional media. 